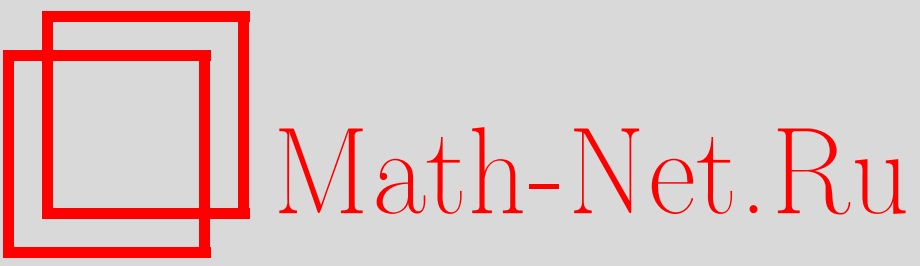

М. П. Бурлаков, Пространства подвижности, Итоги науки и техн. Сер. Соврем. мат. и ее прил. Темат. обз., 2020, том 180, 17-22

DOI: https://doi.org/10.36535/0233-6723-2020-180-17-22

Использование Общероссийского математического портала Math-Net.Ru подразумевает, что вы прочитали и согласны с пользовательским соглашением

http://www.mathnet.ru/rus/agreement

Параметры загрузки:

IP: 54.89 .56 .158

26 апреля 2023 г., 04:50:17 


\title{
ПРОСТРАНСТВА ПОДВИЖНОСТИ
}

\author{
(c) 2020 г. $\quad$ М. П. БУРЛАКОВ
}

\begin{abstract}
АннотАция. В статье рассматриваются пространства, обобщающие пространства Галилея, в которых действует нелинейная группа преобразований. Показано, что такие пространства соответствуют пространству-времени динамики, фундаментальные уравнения которой имеют порядок больше двух.
\end{abstract}

Ключевые слова: пространство Галилея, группа движений, динамическое уравнение.

\section{MOBILITY SPACES}

\section{(c) 2020 M. P. BURLAKOV}

\begin{abstract}
In this paper, we consider spaces generalizing Galileo spaces endowed with an action of a nonlinear transformation group. We prove that such spaces correspond to the space-time of a dynamics whose fundamental equations have order $>2$.
\end{abstract}

Keywords and phrases: Galilean space, motion group, dynamic equation.

AMS Subject Classification: 51P05, 51A06

По всей видимости, Эли Картан первым обратил внимание на то, что группа преобразований Галилея

$$
x^{\prime}=x+x_{0}+u_{1} t, \quad y^{\prime}=y+y_{0}+u_{2} t, \quad z^{\prime}=z+z_{0}+u_{3} t, \quad t^{\prime}=t
$$

порождает в четырехмерном аффинном пространстве некоторую метрическую геометрию, в которой «длина» вектора

$$
\rho=t e_{0}+x e_{1}+y e_{2}+z e_{3} \equiv t e_{0}+r
$$

дается его нулевой координатой (см. [3]). В дальнейшем эту геометрию стали называть геометрией Галилея.

Геометрия Галилея имеет естественную механическую интерпретацию, в которой точки характеризуются положением в трехмерном пространстве и моментом времени. При этом преобразования Галилея взяты так, чтобы дифференциальные уравнения

$$
\frac{d^{2} x}{d t^{2}}=0, \quad \frac{d^{2} y}{d t^{2}}=0, \quad \frac{d^{2} z}{d t^{2}}=0, \quad \text { или } \quad \frac{d^{2} \boldsymbol{r}}{d t^{2}}=0
$$

были инвариантны под действием этих преобразований, что означает независимость свободного движения материальных точек от скорости равномерного и прямолинейного движения системы отсчета. В этом состоит приниип относительности Галилея.

Пространство-время Галилея обобщают пространства подвижности $m$-го порядка $\mathbf{\Pi}_{m}^{n}$, геометрия которых порождена группой преобразований следующего вида: $t^{\prime}=t$ и

$$
x^{\prime k}=x^{k}+b_{0}^{k}+b_{1}^{k} t+b_{2}^{k} t^{2}+\ldots+b_{m-1}^{k} t^{m-1} .
$$


Такую группу мы будем называть группой подвижности $m$-того порядка и обозначать $\mathbf{G}_{m}$. При этом легко видеть, что преобразования этой группы сохраняют дифференциальные уравнения

$$
\frac{d^{m} x^{k}(t)}{d t^{m}}=0
$$

Мы будем также считать, что гиперповерхности $t=$ const всегда представляют собой $n$-мерное евклидово (аффинное) пространство.

Таким образо,м пространства подвижности $m$-того порядка дают геометрическую интерпретацию механики, в которой принцип относительности Галилея расширен до скоростей, порядок которых не превышает $m-1$. Иначе говоря, свободное движение материальных точек в пространствах подвижности $m$-того порядка определяется дифференциальными уравнениями (2).

Интегрируя дифференциальные уравнения (2), мы получим параметрические уравнения траекторий свободного движения материальных частиц (или короче - уравнения свободных траекторий) в пространстве подвижности $m$-того порядка:

$$
x^{k}(t)=u_{0}^{k}+u_{1}^{k} t+u_{2}^{k} t^{2}+\ldots+u_{m-1}^{k} t^{m-1} .
$$

Свободные траектории представляют собой обобщение неособых прямых геометрии Галилея (см. [5]); их свойства аналогичны свойствам неособых прямых пространства Галилея.

В частности, в пространстве Галилея через любые две точки, у которых не совпадают временные координаты, проходит единственная прямая. Аналогичное свойство справедливо и для свободных траекторий. Мы будем называть $m$ точек разделенными, если у них нет совпадающих временных координат. Тогда можно утверждать, что через $m$ разделенных точек $X_{\alpha}\left(x_{\alpha}^{k}, t_{\alpha}\right)$ проходит одна и только одна свободная траектория. Это следует из того, что системы линейных уравнений для отыскания в параметрических уравнениях (3) свободной траектории, проходящей через точки $X_{\alpha}\left(x_{\alpha}^{k}, t_{\alpha}\right)$, коэффициентов $u_{\alpha}^{k}$

$$
u_{0}^{k}+u_{1}^{k} t_{\alpha}+u_{2}^{k} t_{\alpha}^{2}+\ldots+u_{m-1}^{k} t_{\alpha}^{m-1}=x_{\alpha}^{k}
$$

имеют одинаковый определитель

$$
\Delta_{m}(t) \equiv \Delta_{m}\left(t_{1}, t_{2}, \ldots, t_{m}\right)=\left|\begin{array}{ccccc}
t_{m}^{m-1} & t_{m}^{m-2} & \ldots & t_{m} & 1 \\
t_{m-1}^{m-1} & t_{m-1}^{m-2} & \ldots & t_{m-1} & 1 \\
\ldots & \ldots & \ldots & \ldots & \ldots \\
t_{1}^{m-1} & t_{1}^{m-2} & \ldots & t_{1} & 1
\end{array}\right|=\prod_{k>h}\left(t_{k}-t_{h}\right)
$$

отличный от нуля в случае разделенных точек $X_{\alpha}\left(x_{\alpha}^{k}, t_{\alpha}\right)$.

Величина $\Delta_{2}\left(t_{1}, t_{2}\right)=t_{2}-t_{1}$ представляет собой галилеево расстояние между двумя точками $X_{1}\left(t_{1}, x_{1}, y_{1}, z_{1}\right)$ и $X_{2}\left(t_{2}, x_{2}, y_{2}, z_{2}\right)$ пространства-времени Галилея. Тогда определитель $\Delta_{m}\left(t_{1}, t_{2}, \ldots, t_{m}\right)$ можно принять за некоторую «меру близости» $m$ точек в пространстве подвижности $m$-того порядка; мы будем называть эту величину плотностъю кортежа точек $X_{\alpha}\left(x_{\alpha}^{k}, t_{\alpha}\right) \in \Pi_{m}^{n}$, где $\alpha=\overline{1, m}$.

Важнейшим свойством расстояния является аддитивность этой величины. Очевидно, что галилеево расстояние обладает свойством аддитивности, так как $\Delta_{2}\left(t_{1}, t_{2}\right)+\Delta_{2}\left(t_{2}, t_{3}\right)=t_{2}-t_{1}+$ $t_{3}-t_{2}=\Delta_{2}\left(t_{1}, t_{3}\right)$. Своеобразным свойством аддитивности обладает и плотность кортежа точек. Нетрудно видеть, что имеет место тождество:

$$
\Delta_{m}\left(t_{0}, t_{2}, \ldots, t_{m}\right)+\Delta_{m}\left(t_{1}, t_{0}, \ldots, t_{m}\right)+\ldots+\Delta_{m}\left(t_{1}, t_{2}, \ldots, t_{0}\right)=\Delta_{m}\left(t_{1}, t_{2}, \ldots, t_{m}\right) .
$$

Например:

$$
\left|\begin{array}{ccc}
t_{3}^{2} & t_{3} & 1 \\
t_{2}^{2} & t_{2} & 1 \\
t_{0}^{2} & t_{0} & 1
\end{array}\right|+\left|\begin{array}{ccc}
t_{3}^{2} & t_{3} & 1 \\
t_{0}^{2} & t_{0} & 1 \\
t_{1}^{2} & t_{1} & 1
\end{array}\right|+\left|\begin{array}{ccc}
t_{0}^{2} & t_{0} & 1 \\
t_{2}^{2} & t_{2} & 1 \\
t_{1}^{2} & t_{1} & 1
\end{array}\right|=\left|\begin{array}{ccc}
t_{3}^{2} & t_{3} & 1 \\
t_{2}^{2} & t_{2} & 1 \\
t_{1}^{2} & t_{1} & 1
\end{array}\right|
$$


Рассмотрим теперь произвольную линию $l$ с параметрическими уравнениями $x^{k}=x^{k}(t)$ в пространстве подвижности $\boldsymbol{\Pi}_{m}^{n}$ и сконструируем величины $\Delta_{m+1}(x), \Delta_{m+1}(y), \Delta_{m+1}(z)$, где

$$
\Delta_{m+1}\left(x^{k}\right)=\left|\begin{array}{cccccc}
x^{k}\left(t_{m}\right) & t_{m}^{m-1} & t_{m}^{m-2} & \ldots & t_{m} & 1 \\
x^{k}\left(t_{m-1}\right) & t_{m-1}^{m-1} & t_{m-1}^{m-2} & \ldots & t_{m-1} & 1 \\
\ldots & \ldots & \ldots & \ldots & \ldots & \ldots \\
x^{k}\left(t_{1}\right) & t_{1}^{m-1} & t_{1}^{m-2} & \ldots & t_{1} & 1 \\
x^{k}\left(t_{0}\right) & t_{0}^{m-1} & t_{0}^{m-2} & \ldots & t_{0} & 1
\end{array}\right|
$$

и так далее. Нетрудно видеть, что эти величины инвариантны относительно действия группы подвижности.

Каков механический смысл величин $\Delta_{m+1}(x), \Delta_{m+1}(y), \Delta_{m+1}(z) ?$

Мы рассмотрим этот вопрос на модельном примере пространства-времени Галилея. Если мы положим

$$
x(t)=x_{0}+v_{x} t+a_{x} t^{2}+\ldots, \quad y(t)=y_{0}+v_{y} t+a_{y} t^{2}+\ldots, \quad z(t)=z_{0}+v_{z} t+a_{z} t^{2}+\ldots,
$$

TO

$$
\begin{aligned}
& \Delta_{3}(x)=\left|\begin{array}{lll}
x\left(t_{1}\right) & t_{1} & 1 \\
x\left(t_{2}\right) & t_{2} & 1 \\
x\left(t_{3}\right) & t_{3} & 1
\end{array}\right|=\left|\begin{array}{lcc}
x_{0}+v_{x} t_{1}+a_{x} t_{1}^{2}+\ldots & t_{1} & 1 \\
x_{0}+v_{x} t_{2}+a_{x} t_{2}^{2}+\ldots & t_{2} & 1 \\
x_{0}+v_{x} t_{3}+a_{x} t_{3}^{2}+\ldots & t_{3} & 1
\end{array}\right|= \\
& =a_{x}\left(t_{3}-t_{2}\right)\left(t_{3}-t_{1}\right)\left(t_{2}-t_{1}\right)+o\left(\left(t_{3}-t_{2}\right)\left(t_{3}-t_{1}\right)\left(t_{2}-t_{1}\right)\right),
\end{aligned}
$$

и так далее. Таким образом, величины

$$
a_{x}=\lim \frac{\Delta_{3}(x)}{\Delta_{3}(t)}, \quad a_{y}=\lim \frac{\Delta_{3}(y)}{\Delta_{3}(t)}, \quad a_{z}=\lim \frac{\Delta_{3}(z)}{\Delta_{3}(t)},
$$

инвариантные относительно действия группы Галилея, представляют собой значения координат мгновенного ускорения (в начальный момент времени). Аналогичный смысл мгновенной скорости $m$-того порядка имеют и пределы отношений $\Delta_{m+1}\left(x^{k}\right) / \Delta_{m+1}(t)$, инвариантных относительно действия группы подвижности $m$-того порядка.

Изучение пространств подвижности $m$-того порядка представляет интерес как простейший пример геометрии с нелинейной группой движений. Более сложные примеры геометрий с нелинейными группами преобразований получаются если композицию преобразований брать на некотором факторкольце кольца многочленов. Например, если в группе подвижности $m$-того порядка, тривиальное преобразование времени $t^{\prime}=t$ заменить на преобразование вида

$$
t^{\prime}=c_{0}+c_{1} t+c_{2} t^{2}+\ldots+c_{m-1} t^{m-1}
$$

а композицию таких преобразований вычислять не в кольце многочленов $\mathbb{R}[t]$, а в факторкольце $\mathbb{R}[t] /\left(t^{m}\right)$. Нетрудно видеть, что в этом случае пространственная часть группы подвижности сохраняется, а инвариантом будет отношение плотностей кортежей точек, расположенных на свободных траекториях. Можно также отказаться от аффинной структуры сечений постоянного времени. В галилеевом случае это приводит нас к геометрической трактовки ньютоновской теории тяготения (см. [3]). Но мы не будем касаться здесь геометрии таких пространств, так как это уведет нас далеко от темы данной статьи.

Вместо этого мы покажем, что расширение группы Галилея до групп подвижности диктуется некоторыми соображениями теоретической механики, дополненной принципом эквивалентности тяготения и инерции. И это дает нам дополнительный резон для изучения геометрии пространств подвижности.

Как известно, в фундаментальных уравнениях Ньютона

$$
m \frac{d^{2} r(t)}{d t^{2}}=F(t, r(t), v(t))
$$


силовые поля могут зависеть лишь от времени $t$, положения в пространстве $r(t)$ и скорости $v(t)=d r(t) / d t$. По сути дела это утверждение есть просто другая формулировка динамического закона (8). Владимир Игоревич Арнольд формулирует это так: «Начальное состояние механической системы (совокупность положений и скоростей точек системъ в какой-нибудъ момент времени) однозначно определяет все ее движение». И дальше академик замечает: « $М$ з не успеваем удивитъся этому факту, так как узнаем его очень рано. Можно представить себе мир, в котором для определения будущего системы нужно в начальный момент знатъ такље ускорения. Опьт показывает, что наш мир не таков» (см. [1]).

Последнее, впрочем, не совсем верно. На самом деле могут существовать силы, зависящие от ускорения движущегося материального тела. Чтобы показать это, мы обратим внимание читателя на реактивное движение ракеты, которое описывает формула Мещерского (см. [2])

$$
M(t) \frac{d v(t)}{d t}=-u(t) \frac{d m(t)}{d t}+F_{0}
$$

где $M(t)$ - переменная масса ракеты, $v(t)$ - ее скорость, $m(t)$ - масса отбрасываемого вещества реактивной струи, $u(t)$ - скорость частиц реактивной струи, $F_{0}$ - результирующая сторонних сил. Таким образом, реактивная сила $F_{\rho}=-u(t) d m(t) / d t$ зависит от скорости расхода топлива и скорости истечения реактивной струи.

Теперь представим себе космический корабль, снабженный ионными двигателями, в которых скорость истечения реактивной струи зависит от напряженности электрического поля, разгоняющего ионы, которые и образуют реактивную струю. Согласно принципу относительности Галилея, экипаж космического корабля может определить лишь его относителъную скорость, наблюдая какие-либо внешние объекты. Напротив, ускорение космического корабля абсолютное, и может быть определено без привязки к тем или иным внешним ориентирам, так как согласно принципу эквивалентности ускорение будет ощущаться как некоторое гравитационное поле. Таким образом, космический корабль можно снабдить акселерометром, который, если его синхронизировать с электромагнитными ускорителями заряженных частиц, образующих реактивную струю, создаст нам реактивную силу, зависящую от ускорения самого космического корабля.

Существование (в принципе) сил, зависящих от ускорения, приводит нас к динамическому уравнению третъего порядка

$$
\mu \frac{d^{3} r(t)}{d t^{3}}=F(t, r(t), v(t), a(t))
$$

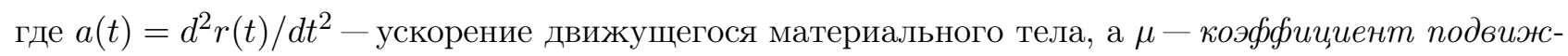
ности, заменяющий в механике третьего порядка массу классической механики. То есть описанный выше мысленный эксперимент с космическим кораблем, ионные двигатели которого синхронизированы с акселерометром, приводит нас к ситуации, упомянутой академиком Арнольдом «Можно представить себе мир, в котором для определения будущего системы нужно в начальный момент знать также ускорения». И так как свободное движение в таком мире дается уравнениями

$$
\frac{d^{3} r(t)}{d t^{3}}=0 \quad \text { или } \quad \frac{d^{3} x(t)}{d t^{3}}=0, \quad \frac{d^{3} y(t)}{d t^{3}}=0, \quad \frac{d^{3} x(t)}{d t^{3}}=0
$$

то пространство-время такого мира будет иметь геометрию пространства подвижности третьего порядка.

Здесь, однако, возникает естественное возражение. В пространстве подвижность третьего порядка свободно движущаяся материальная точка может иметь любое (постоянное) ускорение. В то время как весь наш опыт говорит о том, что если на материальную точку не действуют сторонние силы (или их равнодействующая равна нулю), то материальная точка будет двигаться с нулевым ускорением, т.е. равномерно и прямолинейно. Это и констатирует Арнольд в приведенной выше цитате: «Onыт показывает, что наш мир не таков». 
Впрочем, мы можем устранить противоречие между опытом, говорящим в пользу пространства-времени Галилея и существованием сил, зависящих от ускорения, откуда следует, что пространство-время имеет структуру пространства подвижности третьего порядка. Для этого представим себе, что какая-то обширная область Вселенной заполнена некой средой, которая оказывает сопротивление движению материальных тел с силой, пропорциональной ускорению движущегося материального тела с коэффициентом пропорциональности равным массе этого тела. По сути, эта среда представляет собой физическую причину сил инерции. Эйнштейн называл ее эфиром механики.

«В динамике Ньютона,- пишет Альберт Эйнштейн в своей статье «Об эфире», - „пространство" обладает физической реальностью - в противоположность геометрии и кинематике. Мъ будем называть эту физическую реальность, входящую в закон движения Ньютона „эфиром механики". Появление центробежных сил при вращении тела, материальные точки которого не изменяют взаимных расстояний, показывает, что этот эфир следует понимать не только как некое воображаемое представление теории Нъютона, но что ему соответствует в природе нечто реальное» (см. [2]). И мы (вслед за Эйнштейном) также для краткости будем называть эфиром среду, оказывающую движению материальных тел сопротивление, пропорциональное ускорению.

Посмотрим теперь, как гипотеза «эфира механики» устанавливает согласие между опытом, говорящим в пользу уравнения Ньютона (8) и уравнением (9). Согласно (9) уравнение свободного движения материального тела сквозь эфир, заполняющий некоторую область пространства подвижности третьего порядка, будет иметь вид

$$
\mu \frac{d a(t)}{d t}=-m a(t),
$$

а его решением будет убывающая экспонента

$$
a(t)=a_{0} e^{-m t / \mu},
$$

где $a_{0}$ - начальное ускорение. Отсюда видно, что если на материальное тело не действуют сторонние силы, то каково бы ни было начальное ускорение $a_{0}$, через некоторое время ускорение $a(t)$ станет практически равным нулю, как и должно быть согласно механике Ньютона. Вопрос лишь в том, как быстро уменьшается ускорение $a(t)$, стремясь к нулю? Очевидно, что скорость убывания вектор-функции $a(t)$ зависит от параметра $\tau=\mu / m$, который имеет размерность времени и называется характеристическим временем. (Например, за время $10 \tau \ln 2$ величина ускорения уменьшится более чем в тысячу раз).

Наряду с характеристическим временем целесообразно также ввести время релаксации $T=k \tau$, которое определяется принципиальной возможностью наших приборов регистрировать величину ускорения, т.е. $T$ определяется из условия $a(T)=0$ по показаниям наших приборов, измеряющих ускорение.

Посмотрим теперь, как будет двигаться материальная точка с коэффициентом подвижности $\mu$ и массой $m$ под действием постоянной силы $F_{0}$ и силы сопротивления эфира. В этом случае динамическое уравнение (9) примет вид

$$
\frac{d a(t)}{d t}+\frac{m}{\mu} a(t)=\frac{1}{\mu} F_{0},
$$

а его решением при условии $a(0)=0$ будет вектор-функция

$$
a(t)=\left(1-e^{-m t / \mu}\right) \frac{F_{0}}{m} .
$$

Отсюда видно, что при $t>T$ ускорение материальной точки станет постоянным и равным отношению силы к массе, т.е. для материального тела, движущегося под действием постоянной силы сквозь мировой эфир в пространстве подвижности третьего порядка, мы получим динамическое уравнение Ньютона $m a=F_{0}$. 
А как обстоят дела, когда сила меняется со временем? В этом случае мы будем иметь динамическое уравнение

$$
\frac{d a(t)}{d t}+\frac{m}{\mu} a(t)=\frac{1}{\mu} F(t),
$$

решением которого при $a(0)=0$ будет вектор-функция

$$
a(t)=\frac{e^{-\frac{m}{\mu} t}}{\mu} \int_{0}^{t} F(\lambda) e^{m \lambda / \mu} d \lambda .
$$

Характер поведения этой вектор-функции можно понять, применяя формулу интегрирования по частям. В результате мы получим:

$$
a(t)=\frac{F(t)}{m}-\frac{\dot{F}(t)}{m} \frac{\mu}{m}+\frac{\ddot{F}(t)}{m} \frac{\mu^{2}}{m^{2}}-\ldots-\left(\frac{F(0)}{m}-\frac{\dot{F}(0)}{m} \frac{\mu}{m}+\frac{\ddot{F}(0)}{m} \frac{\mu^{2}}{m^{2}}-\ldots\right) e^{-m t / \mu},
$$

или, учитывая формулу для ряда Маклорена,

$$
a(t)=\frac{1}{m} F(t-\tau)-\frac{1}{m} F(\tau) e^{-t / \tau} .
$$

Формула (15) показывает, что при малом характеристическом времени $\tau$ мы фактически получаем динамическое уравнение Ньютона.

Возникает резонный вопрос: если отклонения динамики материальных тел подчиненной уравнению (9) в доступной нам части Вселенной лежат за пределами возможностей измерительных приборов, то имеет ли «практический» смысл рассматривать такую динамику? Этому есть несколько причин. Во-первых, в динамике третьего порядка сохраняющиеся величины иные, нежели в механике Ньютона, а привычные для нас законы сохранения энергии, импульса и момента импульса имеют лишь приближенный характер. Это в свою очередь приводит к некоторым явлениям, которые в принципе отсутствуют в механике Ньютона. K числу таких явлений, в частности, относятся некоторые антигравитационные эффекты, которые могут проявляться в космологических масштабах.

Последнее замечание относится к релятивистской механике, где считается, что группа Галилея заменяется группой Лоренца. На самом деле уравнения релятивистской механики также инвариантны относительно группы Галилея, только эта группа будет действовать не в четырехмерном аффинном пространстве, а в пятимерном. При этом роль универсального времени Ньютона будет играть собственное время Лоренца, которое мы обозначим $t$, и тогда преобразования Галилея в релятивистском варианте будут иметь такой вид:

$$
x^{\prime \alpha}=x^{\alpha}+u^{\alpha} t,
$$

где $\alpha=0,1,2,3$, т.е. $x^{0}, x^{1}, x^{2}, x^{3}$ - координаты «события» или «мировой точки» в четырехмерном псевдоевклидовом пространстве Минковского, а $u^{0}, u^{1}, u^{2}, u^{3}$ - компоненты четырехмерной скорости. И таким образом мы группу Галилея, действующую в пятимерном пространстве-времени, можем расширить до группы подвижности того или иного порядка.

\section{СПИСОК ЛИТЕРАТУРЫ}

1. Арнольд В. И. Математические методы классической механики. - М.: Наука, 1979.

2. Космодемъянский $A$. A. Теоретическая механика и современная техника. - М.: Просвещение, 1969.

3. Мизнер Ч., Торн К., Уилер Дж. Гравитация. Т. 1. - М.: Мир, 1977.

4. Эйнштейн $A$. Об эфире// в кн.: Собрание научных трудов. - М.: Наука, 1966.

5. Яглом И. М. Принцип относительности Галилея и неевклидова геометрия. - М.: Наука, 1969.

Бурлаков Михаил Петрович

Тверской государственный университет

E-mail: burlakovmihail@mail.ru 